Article

\title{
AAC Double Compression Audio Detection Algorithm Based on the Difference of Scale Factor
}

\author{
Qijuan Huang, Rangding Wang *, Diqun Yan ${ }^{(1)}$ and Jian Zhang \\ Faculty of Electrical Engineering and Computer Science, Ningbo University, Ningbo 315211, China; \\ 18067332664@163.com (Q.H.); yandiqun@nbu.edu.cn (D.Y.); 15757467897@163.com (J.Z.) \\ * Correspondence: wangrangding@nbu.edu.cn
}

Received: 24 May 2018; Accepted: 28 June 2018; Published: 2 July 2018

\begin{abstract}
Audio dual compression detection is an important part of audio forensics. It is of great significance to judge whether the audio has been falsified and forged. This study found that the advanced audio coding (AAC) audio scale factor gradually decreases with the number of compressions increases. Based on this, we propose an AAC double compression audio detection algorithm based on the statistical characteristics of the scale factor difference before and after audio re-compression. The experimental results show that the algorithm can accurately classify dual compressed AAC audio. The average accuracy of AAC audio classification between low-bit-rate transcoding to high-bit-rate is $99.91 \%$, and the accuracy rate between the same bit rate is $97.98 \%$. In addition, experiments with different durations, different noises, and different encoders also proved the better performance of this algorithm.
\end{abstract}

Keywords: AAC audio; dual compression; recompress; scale factor

\section{Introduction}

In the era of mobile Internet [1], the popularization of mobile smart terminals and the continuous advancement of multimedia technology have made great changes in people's daily lives. People can use mobile phones more conveniently to collect photos, audio and share them on the Internet. This multimedia information constantly penetrates into people's lives, making it difficult to distinguish between true and false. In order to detect the authenticity and integrity of multimedia information, multimedia forensics technology has become one of the hot research issues in the field of information security [2].

Digital audio forensics technology is an important part of digital multimedia forensics technology, and audio double compression detection is one of the hot issues in audio forensics technology [3]. Currently on the market for multimedia devices, the audio files are saved in a compressed format. The use of audio editing or processing software to tamper with compressed audio content is accompanied by the creation of double compression. Therefore, audio double compression detection as a previous step in the authenticity assessment of digital content is a necessary condition for judging whether the audio has been falsified or forged [4].

In the MP3 audio double compression detection, Yang et al. [5,6] found that the MP3 audio MDCT coefficients have a significant change in the number of coefficients before and after compression, so that the proportion of the 1 value coefficient in all frequency coefficients is used as a distinguishing feature to realize the effective detection of MP3 audio with low bit rate transcoding to high bit rate. In addition, they studied and analyzed the coefficients of double compressed audio Modified Discrete Cosine Transform (MDCT) coefficients and applied Benford's theorem in image steganalysis to apply to double compressed audio detection. Liu et al. [7] proposed a threshold to achieve single-compressed audio and dual-compressed audio classification by further analyzing the distribution of MDCT coefficients. 
The differences in the distribution of zero-valued coefficients in MDCT coefficients and the difference between smoothness and consistency of non-zero-valued coefficients were analyzed [8,9], which greatly improved the detection accuracy of low bit rate transcoding to high bit rate audio. However, the same bit rate compressed audio detection accuracy still needs to be further improved. T. Bianchi et al. [10,11] introduced the recompression correction principle in the image into the audio. The audio to be measured is decoded, the quantization parameters are extracted, the obtained PCM sample values are clipped and recompressed and coded using the extracted quantization steps. With the use of primary and secondary compressed audio, after the recompression correction, the MDCT coefficient distribution and the pre-correction distribution show different rules, which can effectively detect double-compressed MP3 audio. Ren et al. [12] used the difference in the distribution of Quantized Modified Discrete Cosine Transform (QMDCT) coefficients between recompressed calibration audio and test audio. They performed MP3 recompression audio detection and estimated the compressed audio source rate. In the aspect of AMR recompression audio detection, D Luo et al. $[13,14]$ used a deep learning algorithm and most voting strategies to design decisions to detect double-compressed AMR audio. In their further work, a dual-compression AMR audio detection framework based on the stacked automatic encoder (SAE) network, and the universal background model-Gaussian Mixture Model (UBM-GMM) is proposed.

In advanced audio coding (AAC) audio double compression detection, Jin et al. [15] combined the probability of occurrence of the Huffman code table index with its Markov one-step transition probability and used LIBSVM to classify single and double compressed AAC audio. Its detection rate of compressed audio at the same code rate is low. Li et al. [16] found that the AAC audio frequency components and energy values differ greatly during the compression process and use the Stacked Automatic Encoder (SAE) to detect double-compressed audio.

Through the research, it is found that in the AAC audio compression process, the audio scale factor decreases with the increase of the compression times. Based on the difference in the statistical characteristics of the scale factor before and after audio re-compression, we propose an AAC dual compression audio detection algorithm based on the difference of the scale factor. This algorithm combines the probability of difference of the scale factor and the mean value of the differences together as a detection feature. The LIBSVM is used to classify the recompressed AAC audio. Experiments were performed under different durations, different noises, and different encoders. The results show that the proposed algorithm has high detection accuracy and can effectively detect AAC dual-compressed audio.

\section{AAC Audio Scale Factor Characteristics Change Analysis}

\subsection{Scale Factor in $A A C$}

AAC is an abbreviation of Advanced Audio Coding [17] and is developed based on perceptual audio coding as a basic model. The coding block diagram is shown in Figure 1, and the coding process is as follows. The input PCM signal is time-frequency converted via a filter bank (filter lossless operation). Combined with the psychoacoustic model, the transformed frequency coefficients are quantized and encoded via MDCT processing. Finally, the encoded bit stream is encapsulated to form the final AAC compressed audio.

The AAC encoding is in frames, each frame contains two particles, for two channels each particle contains two channels, for a mono channel each particle contains one channel. Each channel produces 1024 coefficient values after the MDCT transform. AAC has 4 types of transform blocks in MDCT transform: long window block, short window block, start block and end block [18]. The long block is used for a relatively smooth data block in the time domain sample signal, the short block is used for a relatively severe block, and the start block and end block are used as transition block types. It should be noted that in our algorithm, we mainly focus on coding in the case of long window blocks. 
After the MDCT transformation, the most important link is the iterative loop. In the iterative loop, some noise or high frequency information that people cannot hear will be filtered out. The purpose of the inner loop is to adjust the quantization step size, so that the number of bits of the quantized data after Huffman encoding can satisfy the number of bits required by the current encoding parameters. The quantization function in the inner loop of the AAC encoding is

$$
x_{\text {quant }}=\operatorname{int}\left(\left(\left|m d c t_{\text {line }}\right| \times 2^{\frac{1}{4}\left(s f_{\text {decoder }}-100\right)}\right)^{\frac{3}{4}}+0.4054\right)
$$

$\operatorname{int}\left(\right.$ ) is a rounding down function, $s f_{\text {decoder }}$ represents a quantization step size, and mdct $_{\text {line }}, x_{\text {quant }}$ are the spectral coefficients before and after quantization.

Adjusting the quantization step size in audio coding will adjust the scale factor representation range to control the quantization distortion of each scale factor band within the maximum allowable distortion range. The reduction in the scale factor is due in part to the increase in the quantization step size [19]. The high-frequency signal components in the audio have lower energy values. To preserve the accuracy of high-frequency signals, we use smaller quantization steps in the encoding process. After the audio undergoes the compression operation again, the high frequency information is relatively quantized to a value of 0 [20]. Compared with the first compressed audio, the quantization step length is increased, and the scale factor is reduced. By comparing the quantization steps and scale factors of the primary compression and the secondary compression, we believe that the quantization step can reflect the change of each frame of the audio to a certain extent. The scale factor can be used as the feature of AAC double compression audio detection.

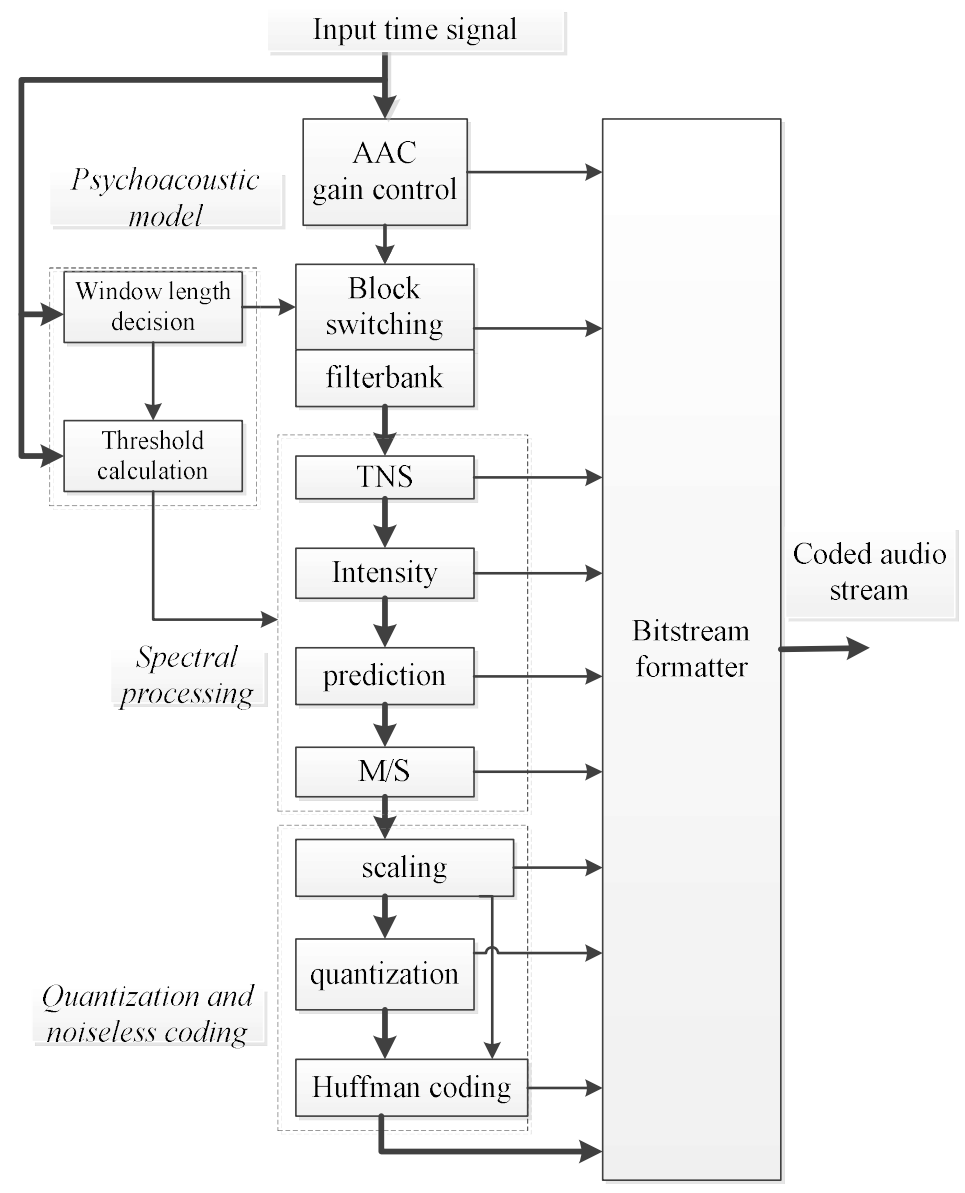

Figure 1. Advanced audio coding (AAC) framework. 


\subsection{Analysis of Changes in the Characteristics of the Scale Factor}

In this algorithm, the default AAC compressed audio has the following two types: (1) low-bit-rate transcoding to high-bit-rate AAC audio, which is often referred to as false-quality audio, and the audio quality of poor audio quality (low-rate) is heavy to form false high-quality (high-rate) audio, and (2) same-bit-rate compressed AAC audio, which is usually an audio tamper recompressed to the original audio format with the same bit rate in order to prevent tampering and other operations from being discovered. For AAC audio with high bit rate transcoding to low bit rate, its meaning in the context of forensics has not been raised, this paper will not discuss. It is worth noting that the AAC compressed audio referred to herein refers only to compressed audio that has experienced two or less times.

To analyze the changes in the AAC audio scale factor, we now randomly select 500 segments of $10 \mathrm{~s}$ of WAV audios including various styles such as classical, rock, folk, and pop. We compressed the 500 segments of WAV audios to get AAC first compression audio at $60 \mathrm{kbps}$ and $150 \mathrm{kbps}$, and then processed the compressed AAC audio to obtain $60 \rightarrow 60 \mathrm{kbps}$ and $60 \rightarrow 150 \mathrm{kbps}$ secondary compressed audio. The decoding and encoding operation of the secondary compressed audio results in third compressed audio of $60 \rightarrow 60 \rightarrow 60 \mathrm{kbps}$ and $60 \rightarrow 150 \rightarrow 150 \mathrm{kbps}$. Now we extract the scale factor of AAC audio for each compression case and count the information. The scale factor for AAC audio is in the range $(0,255)$. The value distribution is approximately Laplacian, and the result is shown in Figure 2. In this algorithm, in order to reduce the experimental dimension, we only take the distribution range of the main values of the scale factor in the statistical analysis $(140,200)$. From Figure 2, we can see that the probability of scale factor decreases with the increase of audio compression times. Through the comparison, it is considered that the AAC double compression audio can be detected by increasing the compression times of the audio to be measured and using the statistical characteristics of the change of the scale factor before and after the audio recompression.

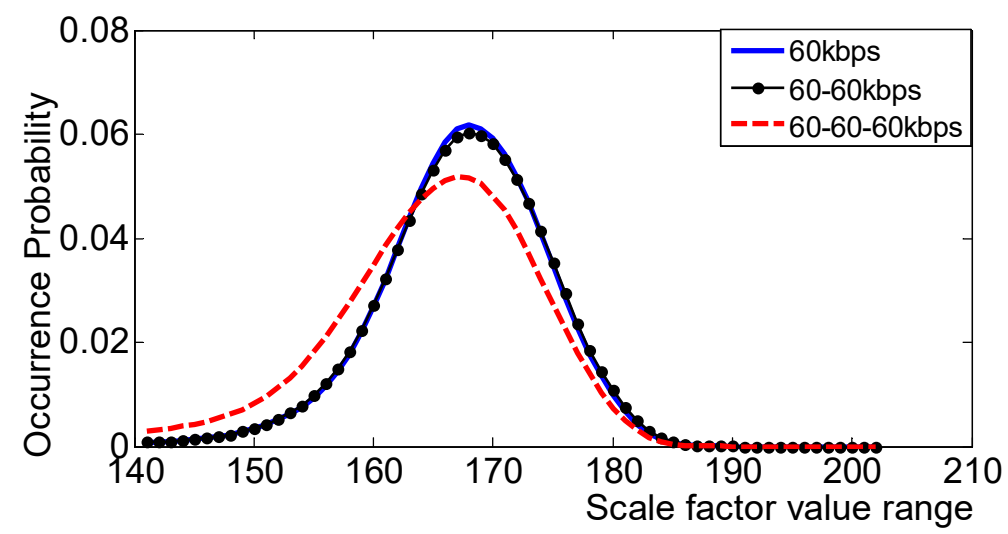

(a) Same-bit-rate compression audio

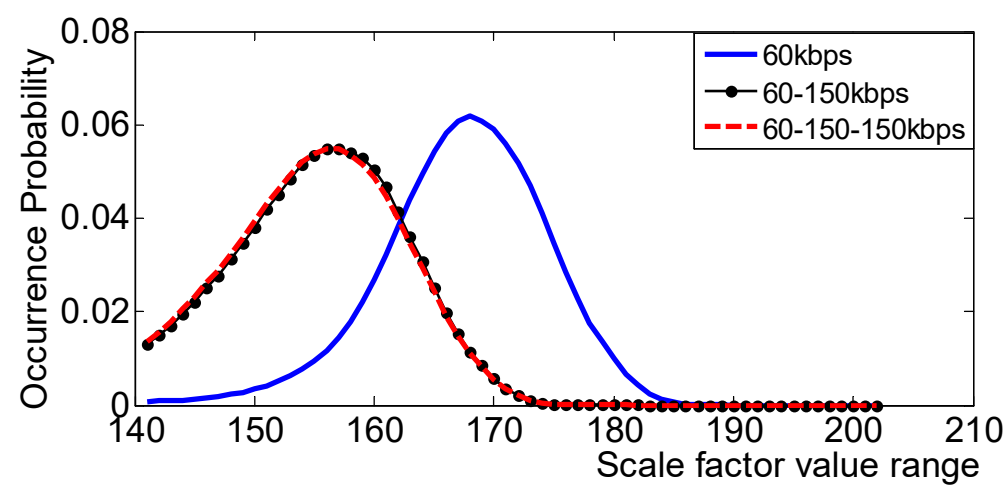

(b)Low-bit-rate transcoding to high-bit-rate audio

Figure 2. AAC audio scale factor occurrence probability. 


\section{Feature Construction and Extraction}

As can be seen from Figure 2, with the increase of compression times, the AAC audio scale factor will be relatively reduced. With this property, the difference between the audio recompressed audio and the original audio scale factor is used as a feature for detecting AAC audio double compression. Figure 3 is a feature extraction flow chart. For the AAC audio to be measured, two operations are performed on it. First, we directly extract the scale factor. Second, the AAC audio to be measured is decompressed, recompressed once with the same compression code rate to obtain the recompressed AAC audio, and the scale factor is re-extracted. The statistical information of the two scale factors is analyzed, and the difference characteristics are used as the final detection features. The AAC audio to be measured and recompressed is denoted as $M 1, M 2$, and the extracted scale factor matrix is denoted as $\mathrm{SF1}$, SF2. The definition is as follows:

$$
\begin{gathered}
S F 1=\left[\begin{array}{ccc}
s f_{1,1} & \cdots & s f_{1, n} \\
\vdots & \ddots & \vdots \\
s f_{m, 1} & \cdots & s f_{m, n}
\end{array}\right] \\
S F 2=\left[\begin{array}{ccc}
s f_{1,1^{\prime}} & \cdots & s f_{1, n^{\prime}} \\
\vdots & \ddots & \vdots \\
s f_{m, 1^{\prime}} & \cdots & s f_{m, n^{\prime}}
\end{array}\right]
\end{gathered}
$$

$n$ represents the number of scale factor bands. If it is a mono $m=2 I$, if it is a dual-channel $m=4 I$, $I$ represents the total number of audio long window frames to be measured.

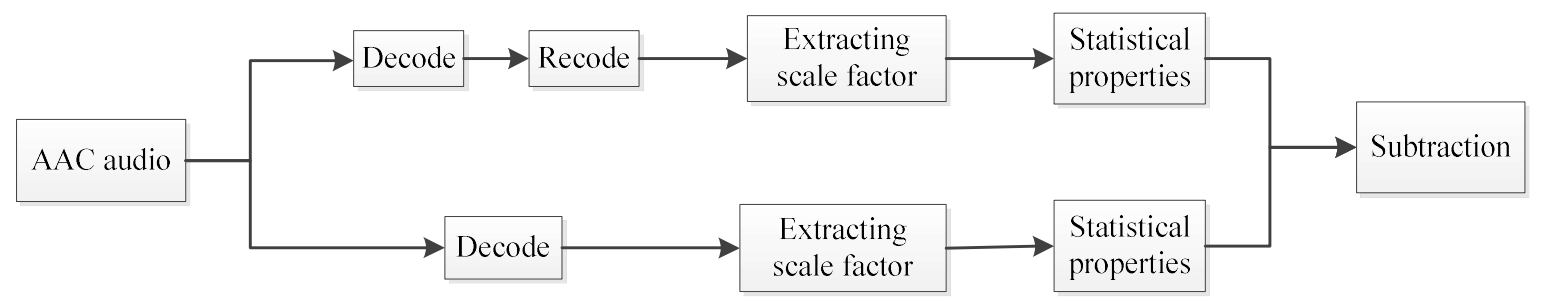

Figure 3. Feature extraction flow chart.

\subsection{Probability Difference Characteristics}

The scale factor decreases as the number of audio compression increases, and its appearance probability will change accordingly as the number of compressions increases. Set the scale factor serial number to $k$. According to the above analysis, the value is mainly concentrated in $(140,200)$, and it is approximately a Laplacian distribution. Let us denote the probabilities of the scale factors of $M 1, M 2$ as $P_{S F 1}, P_{S F 2}$

$$
\begin{aligned}
& P_{S F 1}=\left[P_{S F 1}(k) \mid 140 \leq k \leq 200\right] \\
& P_{S F 2}=\left[P_{S F 2}(k) \mid 140 \leq k \leq 200\right]
\end{aligned}
$$

$P_{S F 1}(k), P_{S F 2}(k)$, respectively, represent the ratio of the number of the scaling factor $k$ in $S F 1, S F 2$ to the total number of scale factors. Then, the probability difference between the scale factor of the test audio and the re-compressed audio is extracted, denoted as $\Delta p(k)$.

$$
\Delta p(k)=P_{S F 2}(k)-P_{S F 1}(k)
$$

For convenience of description, the scale factor probability difference characteristic is denoted as SFPD. Randomly choose 300 segments of $10 \mathrm{~s}$ WAV audios, which include a variety of styles such as classical, rock, folk, popular, and so on. The first compressed AAC audio of $120 \mathrm{kbps}$ is compressed for 
the 300 segments of audios, and the compressed audio is decompressed and recompressed to obtain $120 \rightarrow 135 \mathrm{kbps}$ secondary compressed audio. For the first and second compressed audio, we compress it again at the same code rate. Calculate the scale factor of AAC audio in each case and calculate the difference between the probability of occurrence of the scale factor of the first compressed audio and its recompressed audio $\Delta p_{1}(k)$ and the difference of the probability of occurrence of the scale factor of the second compressed audio and its recompressed audio $\Delta p_{2}(k)$. The Figure 4 is a scatter plot of the probability of occurrence of the scale factor difference $\Delta p_{1}(k), \Delta p_{2}(k)$. In the figure, "o" and " + " respectively represent $\Delta p_{1}(k), \Delta p_{2}(k)$. It can be seen that if the "o" distribution is more discrete, the value is larger, and if the " + " distribution is more concentrated, the value is smaller. The distribution distinction is more obvious, and we think it can be used for AAC double compression audio detection.

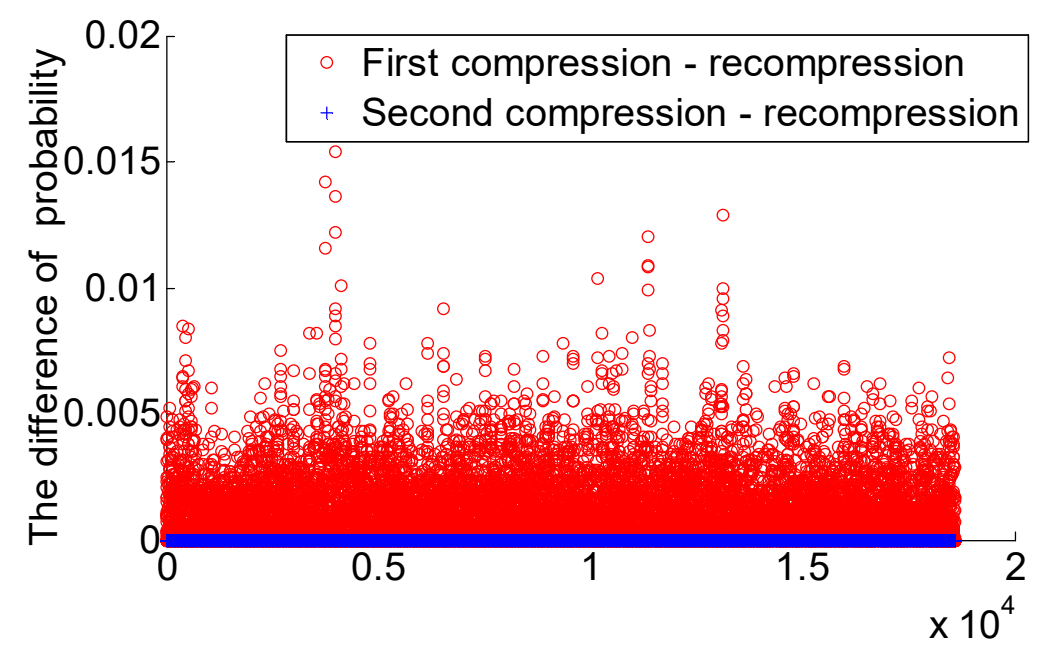

Figure 4. Distribution of probability difference of audio and recompression audio scale factor.

\subsection{Characteristics of Average Difference}

The variation law of the scale factor of the corresponding position after AAC audio first compression and second compression is not obvious. In order to highlight the differences, we extract the mean difference between the current AAC sample and the recompressed audio sample scale factor after decompression in the corresponding frame, which is denoted as $\triangle S F$.

$$
\Delta S F=S F 2-S F 1=\left[\begin{array}{ccc}
\Delta s f_{1,1} & \cdots & \Delta s f_{1, n} \\
\vdots & \ddots & \vdots \\
\Delta s f_{m, 1} & \cdots & \Delta s f_{m, n}
\end{array}\right]
$$

For ease of description, the characteristic of the average value of the scale factor is denoted as SFD. Now we randomly choose 400 segments of audios, including 200 segments of once-compressed audios, re-compressed audio, and 200 segments of secondary-compressed audios, re-compressed audio. The secondary compressed audio includes 100 segments of same-bit-rate compressed audios and 100 segments of low-bit-rate transcoding to high-bit-rate audios. The mean value of the difference between the primary and secondary compressed and recompressed audio scale factors is denoted as $\triangle S F 1, \triangle S F 2$. The Figure 5 shows a scatter plot of 400 segments of audios averages. As can be seen, $\triangle S F 1, \triangle S F 2$ are more distinctive. In particular, the difference is very prominent. For the last 200 segments of AAC audios, the difference between encoding and decoding between the same-bit-rate audio compression is relatively small, the difference performance in Figure 5 is poor, but it can still be distinguished. Through analysis, it is considered that the average value of the scale factor difference 
can reflect the influence of the recompression scale factor globally, which can be used as an effective measure of AAC double compression audio.

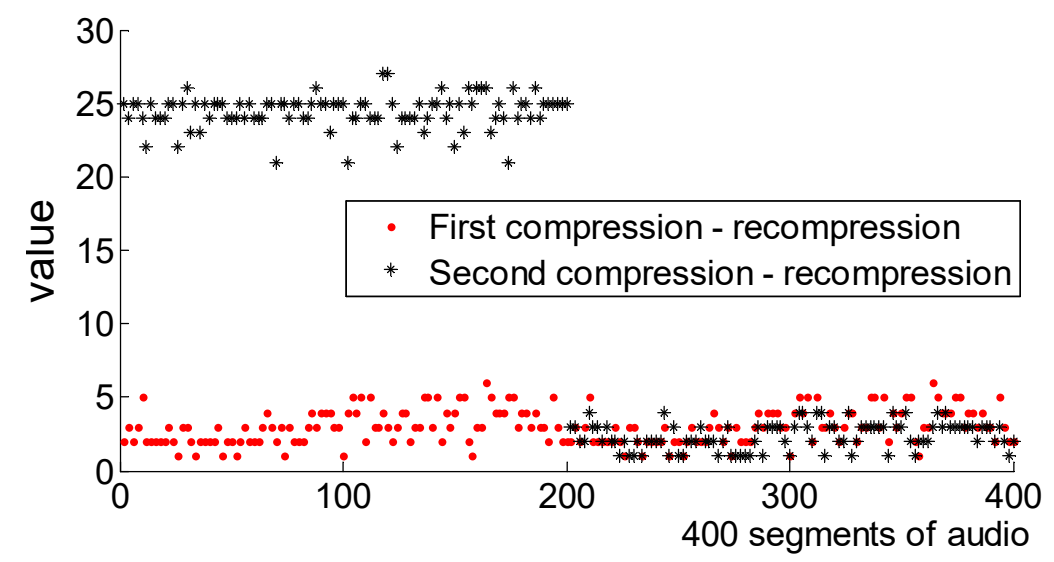

Figure 5. Randomly select the mean of the difference between 400 AAC audio.

\section{Experimental Results and Analysis}

\subsection{Experiment Settings}

We randomly selected 2000 segments of 10-s WAV audios as the original audio sample of this database. This 2000 segments of WAV audio contains various styles, such as blues, pop, classical, country, folk and so on. In this experiment, the AAC codec encodes and decodes audio using the most widely used open source software, FAAC-1.28 and FAAD2-2.7. The first compressed AAC audio is obtained by compressing 2000 segments of WAV audio of the above database using FAAC-1.28. A total of 2000 segments of $(60,75,90,120,135,150)$ kbps were obtained each. Secondary compressed audio is audio that is compressed once by FAAD2-2.7 and then encoded by FAAC-1.28. We have a total of 28 types of secondary compressed audios. For the above first and second compressed audio, the compression operation is performed again at the same-code-rate to obtain a re-compressed audio sample library.

This algorithm selects LIBSVM as a classifier and randomly selects $70 \%$ of the above samples for the training model and the remaining $30 \%$ as a test model. In order to make the test result more accurate, the detection accuracy in the paper is the average of the results after 10 repetitions of the experiment.

\subsection{Feature Set SFPD Feature Detection Results}

Table 1 shows the result of the detection of AAC audio by the SFPD. In the table, BR1 and BR2 indicate the audio compression code rate set in the first and second compression, and the values in the table represent the specific detection accuracy. Taking the $98.33 \%$ value of the seventh row and the eighth column of the table as an example, this value indicates that the method has achieved a combined detection rate of $98.33 \%$ for double-compressed AAC audio that uses $120 \mathrm{kbps}$ and $150 \mathrm{kbps}$ code rate compression (FAAC/FAAD2).

In Table 1, FAAC is an MPEG-4 and MPEG-2 AAC encoder. We can see that the average detection accuracy of low-bit-rate transcoding to high-bit -rate audio is $98.46 \%$, and the same bit rate compressed audio detection is $92.67 \%$. The SFPD achieves better detection results at low-code-rate transcoding to high-code-rate. However, for the same bit rate of compressed audio, the detection rate is relatively low by about 5.79 percentage points, which is due to the relatively small change in the scale factor when the same-bit-rate audio is compressed. When the audio is compressed at the same code rate, the accuracy rate of $(60,75,90) \mathrm{kbps}$ is relatively higher than that when the code rate is $(105,120,135,150)$ kbps. The compressed code rate is high, the loss during compression will be relatively large. We use 
the difference in the probability of the scale factor after the audio recompression. It will reduce the difference between the primary and secondary compression characteristics.

Table 1. SFPD detection accuracy-FAAC (\%).

\begin{tabular}{cccccccc}
\hline \multirow{2}{*}{ BR1 } & \multicolumn{7}{c}{ BR2 } \\
\cline { 2 - 7 } & $\mathbf{6 0}$ & $\mathbf{7 5}$ & $\mathbf{9 0}$ & $\mathbf{1 0 5}$ & $\mathbf{1 2 0}$ & $\mathbf{1 3 5}$ & $\mathbf{1 5 0}$ \\
\hline $\mathbf{6 0}$ & 94.33 & 97.67 & 98.67 & 99 & 97.17 & 99.5 & 97.17 \\
$\mathbf{7 5}$ & & 93.96 & 98.33 & 99 & 97.67 & 99.33 & 98.67 \\
$\mathbf{9 0}$ & & & 94.67 & 97.33 & 98.5 & 99 & 97.83 \\
$\mathbf{1 0 5}$ & & & & 90.33 & 99.5 & 98.67 & 98.76 \\
$\mathbf{1 2 0}$ & & & & & 92.83 & 100 & 98.33 \\
$\mathbf{1 3 5}$ & & & & & & 90.5 & 97.58 \\
$\mathbf{1 5 0}$ & & & & & & & 92.13 \\
\hline
\end{tabular}

\subsection{Feature Set SFD Feature Detection Results}

Table 2 shows the detection result of the SFD for AAC audio. The average detection accuracy of the low-bit-rate transcoding to high-bit-rate AAC audio is $99.3 \%$, and the average accuracy rate between the same bit rate is $95.32 \%$. From the table, we can see that the SFD achieves better detection results at low-bit-rates transcoding to high-bit-rates, and all exceed $98 \%$. Similar to the SFPD, the detection rate is relatively low about 3.98 percentage points for compressed audio at the same code rate. From the comparison between Tables 1 and 2, it can be seen that the SFPD detection accuracy rate is lower than the SFD. It is considered that the AAC audio scale factor gradually decreases with the increase of the number of compressions, and its appearance probability also decreases relatively. It is more intuitive to use the difference of appearance probability of the scale factor to detect without directly using the scale factor difference.

Table 2. SFD detection accuracy-FAAC (\%).

\begin{tabular}{cccccccc}
\hline \multirow{2}{*}{ BR1 } & \multicolumn{7}{c}{ BR2 } \\
\cline { 2 - 8 } & $\mathbf{6 0}$ & $\mathbf{7 5}$ & $\mathbf{9 0}$ & $\mathbf{1 0 5}$ & $\mathbf{1 2 0}$ & $\mathbf{1 3 5}$ & $\mathbf{1 5 0}$ \\
\hline $\mathbf{6 0}$ & 97.45 & 98.75 & 98.5 & 100 & 98.37 & 100 & 98.67 \\
$\mathbf{7 5}$ & & 96.48 & 99.83 & 98.86 & 98 & 100 & 100 \\
$\mathbf{9 0}$ & & & 95.25 & 100 & 100 & 98.68 & 99.33 \\
$\mathbf{1 0 5}$ & & & & 93.73 & 98.75 & 100 & 100 \\
$\mathbf{1 2 0}$ & & & & & 94.5 & 100 & 98.93 \\
$\mathbf{1 3 5}$ & & & & & & 94.75 & 98.71 \\
$\mathbf{1 5 0}$ & & & & & & & 95.13 \\
\hline
\end{tabular}

\subsection{Detection Results of Fusion Feature Sets}

Insufficient detection of the same code rate double compression audio for feature set SFD in the previous section. In combination with the construction method of feature set SFPD in Section 4.2, this section improves the classification performance of the method at the same code rate by adding SFPD statistical features to the SFD. Now we use the different weight ratios of SFPD and SFD, update the fusion weights of different features iteratively, and finally use the fusion weights to classify the output of different feature sets. The iterative process is as follows.

(1) Different feature sets initialize the fusion weight coefficient $W_{i}$

$$
W_{i}=\frac{1}{M}, i=1,2, \ldots M
$$


$M$ is the number of different feature sets, here $M=2, W_{i}=0.5$ during initialization. That is, at the initial iteration, the SFPD and SFD are assigned a weight coefficient of 0.5 .

(2) Iteratively updates $W_{i}$

Assume that the actual tag of $\mathrm{AAC}$ audio is $\operatorname{Lable}(\mathrm{X}), \mathrm{X}=0,1$. Where $\mathrm{X}=1$ means that the sample is a positive sample that is a single compressed AAC audio, and $X=0$ represents that the sample is a negative sample that is double compressed AAC audio. And the classification tag of its feature fusion is identity $(X)$. If $\operatorname{Lable}(X)=$ identity $(X)$, the classification tag of feature fusion is compared with the classification tag of each feature. For sub-features that are judged to be incorrect (that is, the classification tag of feature fusion is inconsistent with the classification tag of sub-feature-SFPD or $S F D$ ), the weight coefficient is subtracted from $\varepsilon$, and the correct sub-feature is judged (that is, the classification tag of the feature fusion is consistent with the classification tag of the sub-feature-SFPD or SFD), with the weight coefficient plus $\varepsilon$. $\varepsilon$ represents the error constant

$$
\varepsilon=\frac{E R R}{N}
$$

$E R R$ indicates the integrated error rate of two sub-features when the classification is detected, and $N$ represents the total number of samples.

Get updated weight coefficient $W_{i}^{*}$

$$
W_{i}^{*}=W_{i} \mp \varepsilon
$$

For the SFPD and SFD initial weight coefficients $W_{1}, W_{2}$ are $0.5,0.5$. For example, one AAC audio sample, the actual tag is Lable $(X)$, and the tagged note of its fusion feature set is identity $(X)$. If Lable $(X)=$ identity $(X)$, we compare the fusion feature set classification results with the SFPD and SFD feature set classification results. Assume that for a certain AAC audio sample, the classification label of the fusion feature set is 1 and the classification label of the sub-feature set $S F P D$ is 0 , and the weight coefficient $W_{1}$ is updated. $W_{1}=W_{1}-\varepsilon$. In this case, in order to maintain the weight coefficient and always be 1 , the weight coefficient $W_{2}$ of the SFD is also updated accordingly, $W_{2}=W_{2}+\varepsilon$.

If $\operatorname{Lable}(X) \neq$ identity $(X)$, discard the sample, perform the above operation on the next sample, and repeat the above steps until all samples have been traversed. The resulting fusion feature set is recorded as Feature fusion

$$
\text { Feature }_{\text {fusion }}=W_{1} * S F P D+W_{2} * S F D
$$

Table 3 shows the detection accuracy of the fusion feature, $W_{1}=0.463, W_{2}=0.537$. The average detection accuracy of low-rate transcoding to high-rate AAC audio is $99.91 \%$, and the average accuracy of same-rate compression detection is $97.98 \%$. Table 3 compares Table 1 with Table 2 and finds that for the detection rate of AAC recompressed audio, the Feature fusion $_{\text {further improves }}$ it. Compared with the SFPD and SFD, the detection accuracy of AAC audio with low bit rate transcoding to high bit rate increased by 1.45 and 0.61 percentage points, respectively. Compared with the SFPD and SFD, the detection accuracy of compression between the same-rate AAC audio increased by 5.31, 2.66 percentage points, respectively. The experimental results show that Feature $_{\text {fusion }}$ integrates SFPD and SFD together well, which makes up for some deficiencies of the sub-feature set in AAC double compression audio detection.

In this paper, the first compressed AAC audio is a positive sample and the second compressed AAC audio is a negative sample. True Positives $(T P)$ indicates that the positive class is positive, True Negatives $(T N)$ indicates that the negative class is negative, False Positives $(F P)$ indicates that the negative class is positive, and False negatives $(F N)$ indicates that the positive class is negative class. 
The data in Table 3 is the accuracy of the test, that is, the ratio of the number of samples correctly classified by the classifier to the total number of samples for a given test data set.

$$
A C C=\frac{T P+T N}{T P+F N+F P+T N}
$$

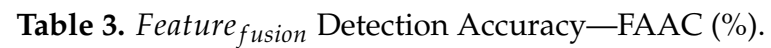

\begin{tabular}{cccccccc}
\hline \multirow{2}{*}{ BR1 } & \multicolumn{7}{c}{ BR2 } \\
\cline { 2 - 8 } & $\mathbf{6 0}$ & $\mathbf{7 5}$ & $\mathbf{9 0}$ & $\mathbf{1 0 5}$ & $\mathbf{1 2 0}$ & $\mathbf{1 3 5}$ & $\mathbf{1 5 0}$ \\
\hline $\mathbf{6 0}$ & 99.03 & 100 & 100 & 100 & 100 & 100 & 100 \\
$\mathbf{7 5}$ & & 98.25 & 100 & 100 & 100 & 100 & 100 \\
$\mathbf{9 0}$ & & & 98.87 & 100 & 100 & 100 & 100 \\
$\mathbf{1 0 5}$ & & & & 96.58 & 100 & 100 & 100 \\
$\mathbf{1 2 0}$ & & & & & 97.83 & 100 & 99.25 \\
$\mathbf{1 3 5}$ & & & & & & 98.03 & 99 \\
$\mathbf{1 5 0}$ & & & & & & & 97.25 \\
\hline
\end{tabular}

The precision $(P)$, which is for the prediction result, indicates that the prediction result is the number of positive samples that have positive samples. There are two possibilities for the prediction. One is to predict the positive class as a positive class $(T P)$ and the other is to predict the negative class as a positive class $(F P)$.

$$
P=\frac{T P}{T P+F P}
$$

The recall $(R)$ indicates the proportion of positive samples in the sample that are predicted correctly. There are two possibilities. One is to predict the positive class as a positive class (TP), and the other is to predict the original positive class as a negative class $(F N)$.

$$
R=\frac{T P}{T P+F N}
$$

The $F \alpha$ value is an evaluation index that combines the precision rate and the recall rate and is used to comprehensively reflect the overall indicators. When $\alpha=1$, it is the most common $F 1$ value.

$$
F \alpha=\left(\alpha^{2}+1\right) \frac{P * R}{P+R}
$$

Table 4 shows the $F 1$ values detected under the scale factor fusion feature set.

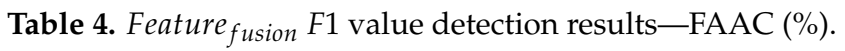

\begin{tabular}{cccccccc}
\hline \multirow{2}{*}{ BR1 } & \multicolumn{7}{c}{ BR2 } \\
\cline { 2 - 7 } & $\mathbf{6 0}$ & $\mathbf{7 5}$ & $\mathbf{9 0}$ & $\mathbf{1 0 5}$ & $\mathbf{1 2 0}$ & $\mathbf{1 3 5}$ & $\mathbf{1 5 0}$ \\
\hline $\mathbf{6 0}$ & 94.63 & 96.58 & 97.63 & 96.87 & 98.57 & 97.25 & 98.54 \\
$\mathbf{7 5}$ & & 93.32 & 98.06 & 95.42 & 96.93 & 98.67 & 95.86 \\
$\mathbf{9 0}$ & & & 94.59 & 97.38 & 98.43 & 96.33 & 97.89 \\
$\mathbf{1 0 5}$ & & & & 93.87 & 97.93 & 98.43 & 98.67 \\
$\mathbf{1 2 0}$ & & & & & 94.02 & 95.67 & 97.33 \\
$\mathbf{1 3 5}$ & & & & & & 95.63 & 96.5 \\
$\mathbf{1 5 0}$ & & & & & & & 92.15 \\
\hline
\end{tabular}




\subsection{Comparison Test}

In order to fully evaluate the performance of the algorithm proposed in this paper, this section reconstructs the features of Jin et al. [15] and conducts comparative experiments. The specific method of Jin feature construction is to use the Huffman code table to use the difference in single compression and double compression of AAC audio and take the probability of occurrence of the Huffman code table index as the first feature. The Markov one-step transition probability is taken as the second feature. The first feature is merged with the second feature and reduced in dimension to obtain the third feature. The LIBSVM is used to classify the single and double compressed AAC audio. Its low bit rate transcoding to high bit rate audio detection rate of $99.72 \%$, the same bit rate audio compression detection rate of $77.38 \%$. Table 5 compares the result of the Jin method with the algorithm of this paper, where " + " indicates that the algorithm detection rate of the algorithm is higher than that of the Jin method detection rate, and "-" indicates that the detection rate of the algorithm is lower than the detection rate of the Jin method. It can be seen from Table 5 that the detection rate of the AAC audio Jin feature with low bit rate transcoding to high bit rate is high, which is not much different from that of the scale factor difference and occurrence probability difference in the algorithm of this paper. However, the detection effect between the same code rate in the literature [15] is poor. The algorithm in this paper solves similar problems and increases the detection rate of the same-rate compressed audio by nearly 20 percentage points. For a better comparison with this algorithm, Table 6 shows the comparison of $F 1$ values between this algorithm and literature [15].

Table 5. Comparison between [15] and the Feature $_{\text {fusion }}$ of this algorithm-FAAC (\%).

\begin{tabular}{cccccccc}
\hline \multirow{2}{*}{ BR1 } & \multicolumn{7}{c}{ BR2 $=$ BR3 } \\
\cline { 2 - 8 } & $\mathbf{6 0}$ & $\mathbf{7 5}$ & $\mathbf{9 0}$ & $\mathbf{1 0 5}$ & $\mathbf{1 2 0}$ & $\mathbf{1 3 5}$ & $\mathbf{1 5 0}$ \\
\hline $\mathbf{6 0}$ & +21.37 & 0 & 0 & 0 & 0 & 0 & 0 \\
$\mathbf{7 5}$ & & +19.85 & 0 & 0 & 0 & 0 & 0 \\
$\mathbf{9 0}$ & & & +23.62 & +0.17 & 0 & 0 & 0 \\
$\mathbf{1 0 5}$ & & & & +25.36 & +1.5 & 0 & 0 \\
$\mathbf{1 2 0}$ & & & & & +20 & +2.33 & +0.1 \\
$\mathbf{1 3 5}$ & & & & & & +18.33 & +0.57 \\
$\mathbf{1 5 0}$ & & & & & & +15.2 \\
\hline
\end{tabular}

Table 6. Comparison between [15] and the Feature $_{\text {fusion }}$ F1 of this algorithm-FAAC (\%).

\begin{tabular}{cccccccc}
\hline \multirow{2}{*}{ BR1 } & \multicolumn{7}{c}{ BR2 $=$ BR3 } \\
\cline { 2 - 8 } & $\mathbf{6 0}$ & $\mathbf{7 5}$ & $\mathbf{9 0}$ & $\mathbf{1 0 5}$ & $\mathbf{1 2 0}$ & $\mathbf{1 3 5}$ & $\mathbf{1 5 0}$ \\
\hline $\mathbf{6 0}$ & +19.89 & +0.13 & -0.21 & +0.05 & -0.12 & +0.34 & +1.33 \\
$\mathbf{7 5}$ & & +20.35 & +0.06 & -0.1 & +0.13 & -0.16 & -2.05 \\
$\mathbf{9 0}$ & & & +20.16 & +0.35 & +1.21 & +0.65 & +0.56 \\
$\mathbf{1 0 5}$ & & & & +18.37 & -1.2 & 0 & -0.06 \\
$\mathbf{1 2 0}$ & & & & & +20.03 & +0.68 & -0.37 \\
$\mathbf{1 3 5}$ & & & & & & +16.98 & -0.31 \\
$\mathbf{1 5 0}$ & & & & & & & +10.59 \\
\hline
\end{tabular}

The AAC and MP3 codec are highly similar in principle. This section also reconstructs the features of the Yang et al. [6] method. It is worth noting that the Yang method is a typical method for detecting MP3 double compression. Its characteristic structure is as follows: the MDCT coefficients of 576 bands of MP3 audio are divided into 32 sub-bands on an average, and each sub-band contains 18 frequency line coefficients. The distribution of the first significant digits of each frequency band coefficient in the first 5, 10, 15 and 20 frequency bands is calculated (1 9). According to the Yang construction characteristics of the idea and the differences between MP3 and AAC, in this section, when reconstructing the Yang method, the first 10 frequency band coefficients are selected, 
and the first significant digits are counted. As a result, a 90-dimensional feature was constructed for each AAC sample. Table 7 shows the accuracy of the modified Yang method for detecting AAC double-compressed audio.

The results in Table 7 show that the detection of AAC double compressed audio has a better detection result in the detection of low-bit-rate transcoding to high-bit-rate recompressed audio. The detection rate at the same-bit-rate compressed audio and $60 \rightarrow 90 \mathrm{kbps}, 60 \rightarrow 75 \mathrm{kbps}$ is lower. This algorithm solves the problem of poor detection rate, and the detection rate is significantly improved. Table 8 shows the comparison of the $F 1$ value of this algorithm compared with the literature [6].

Table 7. Comparison between [6] and the Feature $_{\text {fusion }}$ of this algorithm-FAAC (\%).

\begin{tabular}{cccccccc}
\hline \multirow{2}{*}{ BR1 } & \multicolumn{7}{c}{ BR2 } \\
\cline { 2 - 8 } & $\mathbf{6 0}$ & $\mathbf{7 5}$ & $\mathbf{9 0}$ & $\mathbf{1 0 5}$ & $\mathbf{1 2 0}$ & $\mathbf{1 3 5}$ & $\mathbf{1 5 0}$ \\
\hline $\mathbf{6 0}$ & +24.56 & +30.03 & +25 & +5.03 & 0 & 0 & +0.05 \\
$\mathbf{7 5}$ & & +11.48 & +0.08 & 0 & 0 & 0 & 0 \\
$\mathbf{9 0}$ & & & +20.05 & -0.32 & +0.05 & 0 & +0.1 \\
$\mathbf{1 0 5}$ & & & & +20.97 & +0.54 & +0.22 & +0.07 \\
$\mathbf{1 2 0}$ & & & & & +22.21 & +0.61 & +0.22 \\
$\mathbf{1 3 5}$ & & & & & & +25.95 & +1.43 \\
$\mathbf{1 5 0}$ & & & & & & & +1.44 \\
\hline
\end{tabular}

Table 8. Comparison between [6] and the Feature $_{\text {fusion }}$ F1 of this algorithm-FAAC (\%).

\begin{tabular}{cccccccc}
\hline \multirow{2}{*}{ BR1 } & \multicolumn{7}{c}{ BR2 } \\
\cline { 2 - 8 } & $\mathbf{6 0}$ & $\mathbf{7 5}$ & $\mathbf{9 0}$ & $\mathbf{1 0 5}$ & $\mathbf{1 2 0}$ & $\mathbf{1 3 5}$ & $\mathbf{1 5 0}$ \\
\hline $\mathbf{6 0}$ & +21.35 & +25.63 & +15.68 & +1.56 & -0.32 & +2.03 & -0.11 \\
$\mathbf{7 5}$ & & +12.06 & -0.26 & +0.21 & +0.09 & +1.28 & +0.35 \\
$\mathbf{9 0}$ & & & +18.37 & +0.51 & +0.56 & +0.35 & 0 \\
$\mathbf{1 0 5}$ & & & & +21.35 & -0.05 & -0.24 & -0.21 \\
$\mathbf{1 2 0}$ & & & & & +17.68 & +1.65 & -0.13 \\
$\mathbf{1 3 5}$ & & & & & & +26.35 & +2.35 \\
$\mathbf{1 5 0}$ & & & & & & & +5.68 \\
\hline
\end{tabular}

\subsection{Robust Experiment}

\subsubsection{Different Audio Duration Experiment Results}

In the above experiments, the samples used were all $10 \mathrm{~s}$ AAC audio. To verify the performance of the algorithm in this paper, this section discusses the performance of the Feature $_{\text {fusion }}$ at different times. AAC audio samples of different durations were obtained from 2000 segments of $10 \mathrm{~s}$ WAV audio in the above sample library. We cut out the WAV audio clips of $0.5 \mathrm{~s}, 1 \mathrm{~s}, 2 \mathrm{~s}, 3 \mathrm{~s}, 4 \mathrm{~s}, 5 \mathrm{~s}, 6 \mathrm{~s}, 7 \mathrm{~s}$, $8 \mathrm{~s}$, and $9 \mathrm{~s}$ and reproduced AAC audio sample libraries and re-compressed audio sample libraries of different durations. According to the above experimental process, the features of this paper were extracted, and the Feature fusion $_{\text {was obtained. }}$

Table 9 shows the classification accuracy of the AAC audio double compression with the AAC audio at different durations. The same bit rate compression refers to the recognition rate when the bit rates of single and double compression are the same, and the low bit rate transcoding to high bit rate refers to the recognition rate when the second compression rate is higher than the first compression rate. As the audio duration increases, the detection accuracy of this algorithm also increases, indicating that the algorithm is still valid for AAC audio detection of different durations. 
Table 9. AAC dual compression detection accuracy at different durations-FAAC (\%).

\begin{tabular}{ccc}
\hline Duration (s) & Same-Rate Compression & Low-Bit -Rate Transcoding to High-Bit-Rate \\
\hline $\mathbf{0 . 5}$ & 78.56 & 91.56 \\
$\mathbf{1 . 0}$ & 82.35 & 93.33 \\
$\mathbf{2 . 0}$ & 87.63 & 95.12 \\
$\mathbf{3 . 0}$ & 91.33 & 95.89 \\
$\mathbf{4 . 0}$ & 94.87 & 97.85 \\
$\mathbf{5 . 0}$ & 96.05 & 97.63 \\
$\mathbf{6 . 0}$ & 97.14 & 98.58 \\
$\mathbf{8 . 0}$ & 97.02 & 99.03 \\
$\mathbf{9 . 0}$ & 97.89 & 99.87 \\
$\mathbf{1 0 . 0}$ & 97.98 & 99.91 \\
\hline
\end{tabular}

\subsubsection{Experimental Results under Noise}

In the 4.1 sample setup above, we randomly selected 2000 segments of WAV audio for $10 \mathrm{~s}$. The WAV audio was clean audio. To further verify the robustness of this algorithm, this section discusses the performance of this algorithm in the case of noise addition. We added Gaussian white noise with different signal-to-noise ratios to 2000 original clean audio. We added noise with SNR of $0 \mathrm{~dB}, 10 \mathrm{~dB}, 15 \mathrm{~dB}, 20 \mathrm{~dB}$, and $25 \mathrm{~dB}$ to recreate the sample library and re-compressed audio sample library. According to the above experimental process, the features of this paper were extracted, and the Feature fusion was obtained. Table 10 shows the classification accuracy of AAC audio double compression with the fusion feature of AAC audio at different SNRs. At different signal to noise ratios, the detection accuracy of AAC audio double compression is still high, and when the signal to noise ratio is low, the accuracy rate is higher, indicating that the noise has little impact on the performance of the algorithm to a certain extent. This algorithm has better anti-noise performance.

Table 10. AAC double compression detection accuracy under noise-FAAC (\%).

\begin{tabular}{ccc}
\hline SNR & Same-Rate Compression & Low-Bit-Rate Transcoding to High-Bit-Rate \\
\hline $\mathbf{0 ~ d B}$ & 92.35 & 98.33 \\
$\mathbf{1 0} \mathbf{d B}$ & 91.37 & 98.67 \\
$\mathbf{1 5} \mathbf{d B}$ & 90.05 & 97.45 \\
$\mathbf{2 0} \mathbf{d B}$ & 90.33 & 96.83 \\
$\mathbf{2 5} \mathbf{d B}$ & 87.67 & 95.31 \\
\hline
\end{tabular}

\subsubsection{Different Encoder Experimental Results}

Although the same standard is followed for different encoders, there may be some differences in the specific implementation. To verify that the features selected in this paper are still valid for other encoders, this section selects another popular AAC codec, NeroAACCodec-1.5.1. According to Section 4.1 on the construction of the audio database, NeroAAC's single-compressed audio is obtained by using neroAacEnc (encoder for NeroAACCodec-1.5.1) to compress 2000 segments of $10 \mathrm{~s}$ WAV audio, and then the compressed audio is decompressed by neroAacDec (decoder for NeroAACCodec-1.5.1) and then compressed by neroAacEnc into the corresponding dual-compressed audio. The recompressed audio of once and twice compressed audio is consistent with the above construction method. In the final training and testing, the used classifiers and classifier settings are the same as in Section 4.1.

Table 11 shows the detection accuracy of NeroAAC double compressed audio using the Feature $_{\text {fusion }}$. From the table, we can see that for the AAC audio of different encoders, the Feature $_{\text {fusion }}$ still has a good detection accuracy. The double compressed audio performance of the feature set at low bit rate transcoding to high bit rate is still better than that at the same bit rate compressed audio. From

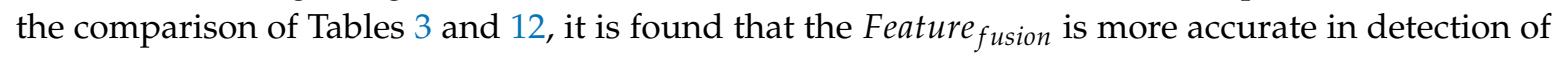


FAAC double compressed audio than in NeroAAC. The analysis found that the change in the scale factor of the NeroAAC audio before and after the first and second compression is smaller than that of the FAAC audio Therefore, the detection rate of the NeroAAC audio is slightly lower when the scale factor feature is used for detection.

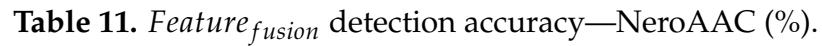

\begin{tabular}{cccccccc}
\hline \multirow{2}{*}{ BR1 } & \multicolumn{7}{c}{ BR2 } \\
\cline { 2 - 8 } & $\mathbf{6 0}$ & $\mathbf{7 5}$ & $\mathbf{9 0}$ & $\mathbf{1 0 5}$ & $\mathbf{1 2 0}$ & $\mathbf{1 3 5}$ & $\mathbf{1 5 0}$ \\
\hline $\mathbf{6 0}$ & 90.13 & 96.3 & $89 . .12$ & 90.45 & 94.65 & 99.3 & 98.27 \\
$\mathbf{7 5}$ & & 80.35 & 85.33 & 89.56 & 96 & 98.98 & 98.32 \\
$\mathbf{9 0}$ & & & 87.47 & 93.56 & 97.9 & 97.03 & 97.66 \\
$\mathbf{1 0 5}$ & & & & 83.22 & 98.67 & 97.35 & 99.27 \\
$\mathbf{1 2 0}$ & & & & & 80.45 & 96.79 & 97.77 \\
$\mathbf{1 3 5}$ & & & & & & 80.65 & 93.56 \\
$\mathbf{1 5 0}$ & & & & & & & 84.33 \\
\hline
\end{tabular}

To further test the validity of the features presented in this algorithm, crossover experiments between different encoders were also performed in this section. That is, the following two cases are (1) compress the 2000 segments of $10 \mathrm{~s}$ WAV audios using FAAC-1.28 to obtain the once compressed audio, use FAAD2-2.7 to decompress it and then use neroAacEnc to compress the corresponding secondary compressed audios and (2) use neroAacEnc to compress 2000 segments of WAV audios to get compressed audios once. Use neroAacDec to decompress and then use FAAC-1.28 to compress the corresponding second compressed audio.

Tables 12 and 13 are the results of crossover experiments in the above two cases. Although the results of the crossover experiments between different encoders are significantly lower than the experimental results of a single encoder, it still has a certain detection effect. For FAAC-NeroAAC crossover experiments, the average recognition accuracy rate of low bit rate transcoding to high bit rate is $91.40 \%$. The average recognition rate of the same-rate audio compression is $81.21 \%$. For NeroAAC-FAAC crossover experiments, the average recognition accuracy of low bit rate transcoding to high bit rate is $88.94 \%$. The average recognition rate of the same-rate audio compression is $76.74 \%$. The Feature $_{\text {fusion }}$ performs relatively well on low bit rate transcoding to high bit rate audio, but it has a large difference in the detection of some AAC double compressed audio. As shown in Table 12, the detection rate of double compressed audio for $60 \rightarrow 135 \mathrm{kbps}$ is $96 \%$, while the detection rate for double compressed audio at $75 \rightarrow 90 \mathrm{kbps}$ is only $83.35 \%$, which is nearly 12.65 percentage points lower. The same is true for Table 13, where the detection rate for double compressed audio at $60 \rightarrow 150 \mathrm{kbps}$ is $94.56 \%$, while the detection rate for double compressed audio at $75 \rightarrow 90 \mathrm{kbps}$ is only $79.59 \%$, which is nearly 14.97 percentage points lower.

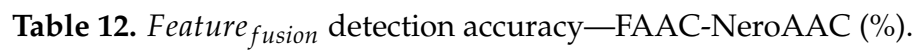

\begin{tabular}{cccccccc}
\hline \multirow{2}{*}{ BR1 } & \multicolumn{7}{c}{ BR2 } \\
\cline { 2 - 8 } & $\mathbf{6 0}$ & $\mathbf{7 5}$ & $\mathbf{9 0}$ & $\mathbf{1 0 5}$ & $\mathbf{1 2 0}$ & $\mathbf{1 3 5}$ & $\mathbf{1 5 0}$ \\
\hline $\mathbf{6 0}$ & 80.45 & 93.17 & 85.57 & 89.65 & 91.16 & 96 & 92 \\
$\mathbf{7 5}$ & & 79.65 & 83.35 & 86.33 & 93.88 & 92.25 & 94.33 \\
$\mathbf{9 0}$ & & & 81.33 & 91.63 & 94.63 & 90.33 & 92.59 \\
$\mathbf{1 0 5}$ & & & & 82.56 & 93.25 & 91.25 & 93.56 \\
$\mathbf{1 2 0}$ & & & & & 79.33 & 89.83 & 90.23 \\
$\mathbf{1 3 5}$ & & & & & & 82.15 & 94.35 \\
$\mathbf{1 5 0}$ & & & & & & & 83 \\
\hline
\end{tabular}




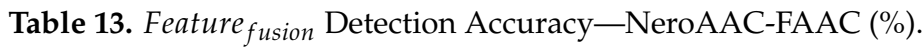

\begin{tabular}{cccccccc}
\hline \multirow{2}{*}{ BR1 } & \multicolumn{7}{c}{ BR2 } \\
\cline { 2 - 8 } & $\mathbf{6 0}$ & $\mathbf{7 5}$ & $\mathbf{9 0}$ & $\mathbf{1 0 5}$ & $\mathbf{1 2 0}$ & $\mathbf{1 3 5}$ & $\mathbf{1 5 0}$ \\
\hline $\mathbf{6 0}$ & 75.33 & 87.67 & 86 & 83.23 & 93.56 & 93.12 & 94.56 \\
$\mathbf{7 5}$ & & 76.45 & 79.59 & 84.58 & 94 & 91.61 & 89.57 \\
$\mathbf{9 0}$ & & & 78.98 & 85.41 & 93.77 & 88.74 & 88.28 \\
$\mathbf{1 0 5}$ & & & & 80 & 90.33 & 90.27 & 84.05 \\
$\mathbf{1 2 0}$ & & & & & 73.56 & 87.47 & 88.68 \\
$\mathbf{1 3 5}$ & & & & & & 74.28 & 83.33 \\
$\mathbf{1 5 0}$ & & & & & & & 78.56 \\
\hline
\end{tabular}

From Tables 3 and 11, Tables 12 and 13, we find that there is a large difference in the detection

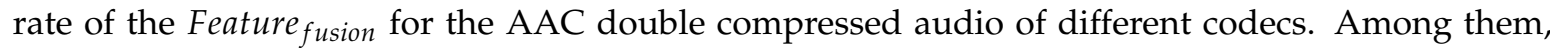
the double-compression audio detection for the FAAC/FAAD2 processing by the codec has higher accuracy. Although the crossover results are lower than those of a single codec, the experimental results indicate that double compressed AAC audio can still be detected.

\section{Conclusions}

In this paper, an AAC double compression audio detection algorithm based on statistical characteristics of scale factor difference is proposed for recompressed AAC audio. By studying the change of the scale factor in the compression process, the double compression AAC audio is classified using the difference feature of the scale factor before and after the audio recompression. Experimental results show that the feature of this paper has better detection performance. The accuracy of AAC audio detection between low code rate transcoding to high code rate is $99.91 \%$, and the detection accuracy rate between audio code compression rate of the same code rate is $97.98 \%$. Experiments with different durations, different noises, and different encoders have proved that the proposed algorithm has strong robustness.

Author Contributions: Conceptualization, R.W. and D.Y.; Methodology, Q.H.; Validation, J.Z.

Funding: This research was funded by the National Natural Science Foundation of China (Grant No. U1736215, 61672302), the Zhejiang Natural Science Foundation (Grant No. LZ15F020002, LY17F020010), the Ningbo Natural Science Foundation (Grant No. 2017A610123), and the Ningbo University Fund (Grant No. XKXL1509, XKXL1503).

Conflicts of Interest: The authors declare no conflict of interest.

\section{References}

1. Li, S.; Xu, L.D.; Zhao, S. 5G Internet of Things: A Survey. J. Ind. Inf. Integr. 2018, 10, 1-9. [CrossRef]

2. Yang, R.; Luo, W.; Huang, J. Multimedia forensics. Sci. Sin. 2013, 43, 1654-1672.

3. Yan, D.; Wang, R.; Zhou, J.; Jin, C.; Wang, Z. Compression history detection for MP3 audio. KSII Trans. Internet Inf. Syst. 2018, 12, 662-675. [CrossRef]

4. Jin, C. Research on Key Technologies of Digital Audio Passive Forensics. Ph.D. Thesis, Ningbo University, Ningbo, China, 2016.

5. Yang, R.; Shi, Y.Q.; Huang, J. Defeating fake-quality MP3. In Proceedings of the 11th ACM Multimedia Security Workshop, MMandSec'09, Princeton, NJ, USA, 7-8 September 2009; pp. 117-124. [CrossRef]

6. Yang, R.; Huang, J. Detecting double compression of audio signal. In Media Forensics and Security II; SPIE: Bellingham, WA, USA, 2010; Volume 7541, p. 75410. [CrossRef]

7. Liu, Q.Z.; Sung, A.H.; Qiao, M.Y. Detection of Double MP3 Compression. Cognit. Comput. 2010, 2, 291-296. [CrossRef]

8. Qiao, M.Y.; Sung, A.H.; Liu, Q.Z. Improved Detection of MP3 Double Compression Using Content-Independent Features. In Proceedings of the IEEE International Conference on Signal Processing, Communication and Computing, Kunming, China, 5-8 August 2013. 
9. Qiao, M.; Sung, A.H.; Liu, Q. Revealing real quality of double compressed MP3 audio. In Proceedings of the ACM Multimedia 2010 International Conference, MM'10, Firenze, Italy, 25-29 October 2010; pp. 1011-1014. [CrossRef]

10. Bianchi, T.; De Rosa, A.; Fontani, M.; Rocciolo, G.; Piva, A. Detection and localization of double compression in MP3 audio tracks. EURASIP J. Inf. Secur. 2014, 2014, 10. [CrossRef]

11. Bianchi, T.; De Rosa, A.; Fontani, M.; Rocciolo, G.; Piva, A. Detection and classification of doublecompressed MP3 audio tracks. In Proceedings of the 2013 ACM Information Hidingand Multimedia Security Workshop, IH and MMSec 2013, Montpellier, France, 17-19 June 2013; pp. 159-164. [CrossRef]

12. Ren, Y.; Fan, M.; Ye, D.; Yang, J.; Wang, L. Detection of double MP3 compression Based on Difference of Calibration Histogram. Multimedia Tools Appl. 2016, 75, 13855-13870. [CrossRef]

13. Luo, D.; Yang, R.; Huang, J. Detecting double compressed AMR audio using deep learning. In Proceedings of the IEEE International Conference on Acoustics, Speech and Signal Processing, Florence, Italy, 4-9 May 2014; pp. 2669-2673. [CrossRef]

14. Luo, D.; Yang, R.; Li, B.; Huang, J. Detection of Double Compressed AMR Audio Using Stacked Autoencoder. IEEE Trans. Inf. Forensics Secur. 2017, 12, 432-444. [CrossRef]

15. Jin, C.; Wang, R.; Yan, D.; Ma, P.; Zhou, J. An efficient algorithm for double compressed AAC audio detection. Multimedia Tools Appl. 2016, 75, 4815-4832. [CrossRef]

16. Li, X. Research on AMR and AAC Audio Dual Compression Detection. Ph.D. Thesis, South China University of Technology, Guangzhou, China, 2015.

17. Institution, B.S. ISO/IEC 13838-7/FPDAM 1. Information Technology. Generic Coding of Moving Pictures and Associated Audio Information. Part 7: Advanced Audio Coding (AAC). Amendment 1: Signalling of Bandwidth Extension.

18. Han, S.; Fingscheidt, T. Robust MPEG-4 high-efficiency AAC with fixed- and variable-length soft-decision decoding. In Proceedings of the 139th Audio Engineering Society International Convention, AES 2015, New York, NY, USA, 29 October-1 November 2015; p. 9399.

19. Gao, Z.; Wei, G. The core technology of the broadband MP3 audio compression. Electroacoust. Technol. 2000, 9, 9-13.

20. D'Alessandro, B.; Shi, Y.Q. MP3 bit rate quality detection through frequency spectrum analysis. In Proceedings of the 11th ACM Workshop on Multimedia and Security, Princeton, NJ, USA, 7-8 September 2009; pp. 57-61. [CrossRef]

(C) 2018 by the authors. Licensee MDPI, Basel, Switzerland. This article is an open access article distributed under the terms and conditions of the Creative Commons Attribution (CC BY) license (http:/ / creativecommons.org/licenses/by/4.0/). 